\title{
Induced Representations of Compact Lie Groups and the Adams Operations
}

\author{
By \\ Akira Kono*
}

\section{§1. Introduction}

Let $\boldsymbol{G}$ be a compact Lie group and $R(\mathbb{G})$ its (complex) representation ring. Then for any element $x \in R(\boldsymbol{G})$ there are complex representations $V$ and $W$ such that $x=V-W$. Then the virtual character $\chi_{x}(g)(g \in \mathbb{G})$ is defined by

$$
\chi_{x}(g)=\chi_{V}(g)-\chi_{W}(g) .
$$

As is well known $x=y$ in $R(\boldsymbol{G})$ if and only if

$$
\chi_{x}(g)=\chi_{y}(g)
$$

for any element $g \in \boldsymbol{G}$.

The Adams operation

$$
\psi^{k}: R(\mathbb{G}) \longrightarrow R(\mathbb{G})
$$

is a ring homomorphism such that

$$
\chi_{\psi^{k}(x)}(g)=\chi_{x}\left(g^{k}\right)
$$

for any $g \in \boldsymbol{G}$ (cf. Adams [1]). On the other hand let $\mathbb{H}$ be a closed subgroup of G. Then Segal defined in [8] the induction homomorphism

$$
\operatorname{Ind}_{\boldsymbol{H}}^{\boldsymbol{G}}: R(\boldsymbol{H}) \longrightarrow R(\boldsymbol{G})
$$

which is characterized by

$$
\chi_{\operatorname{Ind}_{\boldsymbol{H}} \boldsymbol{G}(\boldsymbol{x})}(g)=\sum_{\boldsymbol{a} \boldsymbol{H} \in(\boldsymbol{G} / \boldsymbol{H})} \chi_{x}\left(a^{-1} g a\right)
$$

for generic $g$ where $(\boldsymbol{G} / \boldsymbol{H})^{g}=\{a \mathbb{H} \in \mathbb{G} / \mathbb{H} ; g a \mathbb{H}=a \mathbb{H}\}$ (cf. §2). (In [8], Ind $\boldsymbol{H}_{\boldsymbol{H}}^{\boldsymbol{G}}$ is denoted by $i_{!}$).

Communicated by N. Shimada, August 2, 1980.

* Department of Mathematics, Kyoto University, Kyoto 606, Japan.

This research was partially supported by the Grant-in-Aid for Scientific Research, (No. 574023), Ministry of Education. 
In general $\psi^{k} \circ \operatorname{Ind}_{\boldsymbol{H}}^{\boldsymbol{G}} \neq \operatorname{Ind}_{\boldsymbol{H}}^{\boldsymbol{C}} \circ \psi^{k}$. The purpose of this paper is to show

Theorem 1. If $\left(\left|\boldsymbol{G} / \mathbb{G}^{\circ}\right|, k\right)=1$ then

$$
\psi^{k \circ \operatorname{Ind}} \boldsymbol{G}=\operatorname{Ind}_{\boldsymbol{H}}^{\boldsymbol{G}} \circ \psi^{k}
$$

for any closed subgroup $\mathbb{H}$ of $\mathbf{G}$ where $\boldsymbol{G}^{0}$ is the connected component of the identity of $\mathbf{G}$.

The above theorem plays an important role in the proof of the Adams conjecture in [6].

Let $\boldsymbol{G}$ be a compact connected Lie group such that $\pi_{1}(\boldsymbol{G})$ is torsion free and $\mathbb{U}$ and $\boldsymbol{U}^{\prime}$ be its closed subgroups such that $\boldsymbol{U}^{\prime} \subset \boldsymbol{U}$. Consider the BeckerGottlieb transfer

$$
p_{!}: K\left(\boldsymbol{G} / \boldsymbol{U}^{\prime}\right) \longrightarrow K(\boldsymbol{G} / \boldsymbol{U})
$$

for the fibre bundle $\boldsymbol{U} / \boldsymbol{U}^{\prime} \rightarrow \boldsymbol{G} / \boldsymbol{U}^{\prime} \stackrel{p}{\rightarrow} \boldsymbol{G} / \boldsymbol{U}$ (cf. [5], [7]). Then as a corollary of the above theorem, we have

Corollary 2. If $\boldsymbol{U}$ and $\mathbb{U}^{\prime}$ are connected and of maximal rank, then $p ! \circ \psi^{k}=\psi^{k} \circ p_{!}$.

\section{§. Proof of Theorem 1}

In this section $\boldsymbol{G}$ is a compact Lie group and $k$ is an integer such that $\left(\left|\boldsymbol{G} / \boldsymbol{G}^{0}\right|, k\right)=1$. A closed cyclic subgroup $C$ of $\boldsymbol{G}$ is called a Cartan subgroup if and only if $N_{\boldsymbol{G}}(C) / C$ is a finite group where $N_{\boldsymbol{G}}(C)$ is the normalizer of $C$ in $\boldsymbol{G}$. An element $g \in \mathbb{G}$ is called generic if and only if it is a generator of a Cartan subgroup of $\boldsymbol{G}$. Then generic elements are dense in $\mathbb{G}$ (for details, see Segal [8]). Moreover the following is remarked in Section 1 of Segal [8].

Lemma 2.1. $\left|C / C^{0}\right|$ divides $\left|\boldsymbol{G} / \boldsymbol{G}^{0}\right|^{2}$.

Let $\boldsymbol{H}$ be a closed subgroup of $\boldsymbol{G}$ then the following is also due to Segal [8]:

Lemma 2.2. If $g$ is generic then

$$
\chi_{\operatorname{Ind} \underset{\boldsymbol{H}}{\boldsymbol{G}}(x)}(g)=\sum_{\boldsymbol{a} \in(\boldsymbol{G} / \boldsymbol{H})^{g}} \chi_{x}\left(a^{-1} g a\right),
$$

where $(\boldsymbol{G} / \boldsymbol{H})^{g}=\{a \boldsymbol{H} \in \boldsymbol{G} / \boldsymbol{H} ; g a \boldsymbol{H}=a \boldsymbol{H}\}$.

Using Lemma 2.1, we can easily prove the following:

Lemma 2.3. If $g$ is a generator of a Cartan subgroup $C$ of $\boldsymbol{G}$, then $g^{k}$ 
is also a generator of $C$.

Let $g$ be a generic element. Then $a \mathbb{H} \in(\mathbb{G} / \mathbb{H})^{g}$ if and only if $a^{-1} g a \in \mathbb{H}$ which is equivalent to $a^{-1} C a \subset \mathbb{H}$. Quite similarly $a \mathbb{H} \in(\mathbb{G} / \mathbb{H})^{g^{k}}$ is equivalent to $a^{-1} C a \subset \mathbb{H}$ by Lemma 2.3. So we have

Lemma 2.4. If $g$ is generic then

$$
(\boldsymbol{G} / \mathbf{H})^{g}=(\boldsymbol{G} / \boldsymbol{H})^{g^{h}}
$$

for any closed subgroup $\boldsymbol{H}$ of $\boldsymbol{G}$.

Now we can prove Theorem 1. If $g$ is generic then by Lemma 2.2,

$$
\begin{aligned}
\chi_{\operatorname{Ind}}^{\boldsymbol{G}_{\boldsymbol{H}} \circ \psi^{k}(x)}(g) & =\sum_{a \boldsymbol{H} \in(\boldsymbol{G} / \boldsymbol{H})^{g}} \chi_{\psi^{k}(x)}\left(a^{-1} g a\right) \\
& =\sum_{a \boldsymbol{H} \in(\boldsymbol{G} / \boldsymbol{H})^{g}} \chi_{x}\left(a^{-1} g^{k} a\right)
\end{aligned}
$$

and

$$
\begin{aligned}
\chi_{\psi^{k} \boldsymbol{I I n d}_{\boldsymbol{H}} \boldsymbol{G}(x)}(g) & =\chi_{\mathbf{I n d}_{\boldsymbol{H}}^{\boldsymbol{G}}(x)}\left(g^{k}\right) \\
& =\sum_{a \boldsymbol{H} \in(\boldsymbol{G} / \boldsymbol{H}) g^{k}} \chi_{x}\left(a^{-1} g^{k} a\right),
\end{aligned}
$$

since $g^{k}$ is also generic by Lemma 2.3. Then applying Lemma 2.4, we have

$$
\chi_{\operatorname{Ind}_{H}^{G} \circ \psi^{k}(x)}(g)=\chi_{\psi^{*} \circ \operatorname{Ind} d_{H}^{G}(x)}(g)
$$

for generic $g$. But since virtual characters are continuous and generic elements are dense in $\boldsymbol{G}$, we have

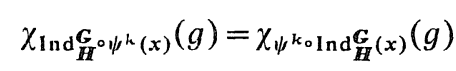

for any $g \in \boldsymbol{G}$ and Theorem 1 is proved.

\section{§3. Proof of Corollary 2}

In this section $\boldsymbol{G}$ is a compact connected Lie group such that $\pi_{1}(\mathbb{G})$ is torsion free. $U$ and $U^{\prime}$ are its closed connected subgroups of maximal rank such that $U^{\prime} \subset \mathbb{U}$.

Let $V$ be a complex representation of $U$. The correspondence $V \rightarrow \mathbb{G} \times{ }_{\mathbb{U}} V$ defines a homomorphism

$$
\alpha=\alpha(G ; U): R(\mathbb{U}) \longrightarrow K(\mathbb{G} / \mathbb{U})
$$

Since $\mathbb{G}$ is a compact free $\mathbb{U}$-space, the following diagram commutes by Proposition 5.4 of [7]: 


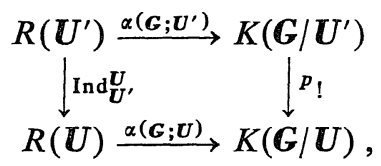

where $p_{!}$is the Becker-Gottlieb transfer for $p: \boldsymbol{G} / \boldsymbol{U}^{\prime} \rightarrow \boldsymbol{G} / \boldsymbol{U}$.

On the other hand if $\boldsymbol{U}^{\prime}$ is connected and of maximal rank then $\alpha\left(\boldsymbol{G} ; \boldsymbol{U}^{\prime}\right)$ is surjective by the Atiyah-Hirzebruch conjecture (cf. Snaith [9] or Pittie [10]). So to prove $p_{!} \circ \psi^{k}=\psi^{k} \circ p_{!}$, we need only show $p_{!} \circ \psi^{k} \circ \alpha\left(\boldsymbol{G} ; \boldsymbol{U}^{\prime}\right)=\psi^{k} \circ p_{!} \circ \alpha\left(\boldsymbol{G} ; \boldsymbol{U}^{\prime}\right)$. Since $\alpha\left(\boldsymbol{G} ; \boldsymbol{U}^{\prime}\right) \circ \psi^{k}=\psi^{k} \circ \alpha(\boldsymbol{G} ; \boldsymbol{U})$ by definitions and Ind $\boldsymbol{U}^{\prime} \circ \psi^{k}=\psi^{k} \circ$ Ind $_{\boldsymbol{U}^{\prime}}^{\boldsymbol{U}}$, by Theorem 1 , we have

$$
\begin{aligned}
p_{!} \circ \psi^{k} \circ \alpha\left(\boldsymbol{G} ; \boldsymbol{U}^{\prime}\right) & =p ! \circ \alpha\left(\boldsymbol{G} ; \boldsymbol{U}^{\prime}\right) \circ \psi^{k} \\
& =\alpha(\boldsymbol{G} ; \boldsymbol{U}) \circ \operatorname{Ind}_{\boldsymbol{U}^{\prime}}^{\boldsymbol{U}} \circ \psi^{k} \\
& =\alpha(\boldsymbol{G} ; \boldsymbol{U}) \circ \psi^{k} \circ \operatorname{Ind}_{U^{\prime}} \\
& =\psi^{k} \alpha(\boldsymbol{G} ; \boldsymbol{U}) \circ \operatorname{Ind}_{\boldsymbol{U}^{\prime}}^{\boldsymbol{U}} \\
& =\psi^{k \circ p} \circ \propto \alpha\left(\boldsymbol{G} ; \boldsymbol{U}^{\prime}\right)
\end{aligned}
$$

and so Corollary 2 is obtained.

\section{References}

[1] Adams, J. F., Vector fields on spheres, Ann. Math., 75 (1962), 603-632.

[2] — Lectures on Lie groups, Benjamin, 1969.

[3] Atiyah, M. F., K-theory, Benjamin, 1967.

[4] Atiyah, M. F. and Hirzebruch, F., Vector bundles and homogeneous spaces, Proc. Symp. Pure Math., 3 (1961), 7-38.

[ 5 ] Becker, J. C. and Gottlieb, D. H., The transfer map and fibre bundles, Topology, 14 (1975), 1-12.

[6] Kono, A., On the order of certain elements of $J(x)$ and the Adams conjecture, Publ. RIMS, Kyoto Univ., 17 (1981), 557-564.

[7] Nishida, G., The transfer homomorphism in equivariant generalized cohomology theories, J. Math. Kyoto Univ., 18 (1978), 435-451.

[ 8 ] Segal, G. B., The representation ring of a compact Lie group, Publ. I.H.E.S., 34 (1968), 113-128.

[9] Snaith, V.P., On the K-theory of homogeneous spaces and the conjugate bundles of Lie groups Proc. London Math. Soc., 22 (1971), 562-584.

[10] Pittie, H. V., Homogeneous vector bundles on homogeneous spaces, Topology, 11 (1972), 199-203. 